\title{
Development of Software for Calibration and Testing of Geometric Errors of Low Cost Desktop Publishing (DTP) Scanners
}

\author{
Dr. Khalid L. A. El-Ashmawy \\ Department of Civil Engineering \\ College of Engineering and Islamic Architecture \\ Umm Al-Qura University \\ Makkah \\ Saudi Arabia
}

\begin{abstract}
Scanners have been used as input devices in photogrammetric and cartographic applications mainly for the digitization of aerial images and maps. Photogrammetric scanners are expensive while DeskTop Publishing (DTP) scanners have low prices, and may have good performance and sufficient resolution for the required applications. There are two types of DTP scanners: low-cost DTP scanners and high-cost DTP scanners. The main disadvantage of low-cost DTP scanners is the lack of geometric accuracy and stability. In addition, the scanner vendors rarely provide the users with software for accuracy testing and calibration.

This paper emphasizes developing software, called ScanCal, for testing the geometric accuracy and calibration of DTP scanners. An overview of the development and capabilities of the ScanCal software platform based on the Windows system is presented. The developed software was used for testing the geometric accuracy of low-cost DTP scanners. The results show that the low-cost DTP scanners are unsuitable for photogrammetric applications but may be used for GIS-related applications. Furthermore, the results of the developed calibration method show that a geometric accuracy of 4-6 can be achieved. The achieved accuracy opens the door-way for using low-cost DTP scanners for photogrammetric applications.
\end{abstract}

Key Words: DTP Scanners, Geometric Accuracy, Digital Photogrammetry, Calibration of DTP Scanners, GISrelated Applications.

\section{INTRODUCTION}

Scanners are an essential component in photogrammetric and cartographic applications. They have been used for scanning aerial and satellite images, as well as digitizing topographic and thematic maps, plans, charts and atlases.

Aerial and satellite imageries have been used to derive Digital Terrain Model (DTM), orthoimages, and digital maps. A new trend is the use of digital or orthoimages for generation and updating of databases, generation of orthoimages maps, integration with other raster and vector data and visualization.

Scanned topographic maps have been used as a central base layer within GIS, as a backdrop in different applications, e.g. navigation systems, for visualization, or for subsequent vectorisation of digital map data.

Scanners can be classified as [6]:

- Photogrammetric Quality Scanners or Photogrammetric Scanners: These scanners are able to produce a geometric accuracy of 2-5 $\mu \mathrm{m}$ (for high accuracy applications) typically at resolution up to and over $2000 \mathrm{dpi}$ (dots per inch). These scanners start at a price of about $\$ 45,000$ US.

- Desktop Publishing (DTP) Scanners: A desktop scanner is a less expensive scanner that can be bought or ordered locally. DTP scanners have been developed for applications totally different from the photogrammetric ones. However, since they constitute the largest sector in the scanner market, they are subjected to rapid development and improvements. There are two types of DTP scanners [1]:

- A low cost DTP scanner: It is used typically for house hold use and its price is $\$ 80$ - $\$ 300$ US. 
- A high cost DTP scanner: It is used in graphic arts and publishing houses. Its price is about $\$ 2000-\$ 5000$ US.

DTP scanners [3] typically employ one or more linear CCDs, and move in direction vertical to the CCD to scan a document in one swath. Usually the stage is stationary, and the sensor/optics/illumination move. They can scan binary, halftone, grey level and colour data, may have good and cheap software for setting the scanner parameters, image processing and editing, and can be connected to many computer platforms (such as Macs, PCs and UNIX workstations) via standard interfaces. They can usually scan A4 format, but some can scan up to A3 or even larger size.

DTP scanners have a resolution up to 1200 dpi (21 $\mu m$ pixel size) over the whole scan width. Few scanners offer the option to increase the resolution by projecting a document portion (smaller than the full width) on the CCD.

DTP scanners with automatic density control and user definable tone curves that can be applied during scanning need for the setting of the scan parameters a few minutes as compared to much more time required by most photogrammetric scanners. In particular, the sensor chip and the electronics of DTP scanners are updated faster and are in most cases more modern than the respective parts of photogrammetric scanners.

The major errors of DTP scanners [3] are geometric mechanical positioning inaccuracies, lens distortions, electronic noise and small dynamic range, colour balance and colour misregistration. Other errors can occur depending on the design, construction, and parts of each individual scanner. Whether some errors are slowly or frequently varying depends on the quality and stability of the scanner, e.g. in DTP scanners the positioning errors vary from scan to scan or even within one scan. In DTP scanners, the geometric errors in CCD direction considerably increase towards the borders of the scanner stage, and in scanning direction they may increase slightly towards the end of the scan.

Furthermore, the main disadvantage of DTP scanners [3] is the lack of geometric testing or calibration software. For such testing and calibration procedures softwares should ideally be supplied by the scanner vendors but this is unfortunately a rare case. In addition, the scanner vendors rarely provide the users with all relevant technical specifications of the scanner and with error specifications, e.g. tolerances for the RMSE (Root Mean Square Error) and maximum error that can occur in different cases. Furthermore, geometric testing of the available scanner should be applied by the user periodically to test its performance.

This paper focuses on the following:

- Developing software (called ScanCal) for testing the geometric accuracy and performing geometric calibration of DTP scanners.

- Using the developed software for testing and correcting the geometric errors of low cost DTP scanners.

\section{2- CONCEPT OF SCANCAL SOFTWARE}

The software has been implemented using Visual C++ Compiler V6.0 [10],[13] and designed to be flexible and portable to 32-bit Windows platforms.

The ScanCal software initializes and terminates the operations of five menus, as depicted in Figure 1, namely Grid Generation, Coordinates Extraction, Geometric Accuracy, Scanner Calibration and Calibrating New Scan.

\subsection{Grid Generation Menu}

The main functions of Grid Generation menu are:

- Generating grids, in the form of separate crosses, according to the specified scanner size, spacing and cross size.

- Automatic generation of the reference $\mathrm{x}, \mathrm{y}$ coordinates of the crosses centers and storing these coordinates in specified ASCII file format for later use.

- $\quad$ Saving the generated grids in DXF (Drawing eXchange Format) file format to be plotted using suitable DXF compatible software (e. g. AutoCAD software [1]). The grids should be plotted on high quality paper or special type transparency.

\section{2-2 Coordinates Extraction menu}

This menu reads the scanned file and extracts automatically the $\mathrm{x}$ and $\mathrm{y}$ image coordinates of the grid crosses as follows:

- Transforming the scanned image of grid crosses to black and white image type (this step can be performed during the scanning process). 
International Journal of Advances in Scientific Research and Engineering (ijasre), Vol 8 (1), January -2022

- Searching for the pixels (in black color) for each cross and recording their $\mathrm{x}, \mathrm{y}$ image coordinates (in pixel).

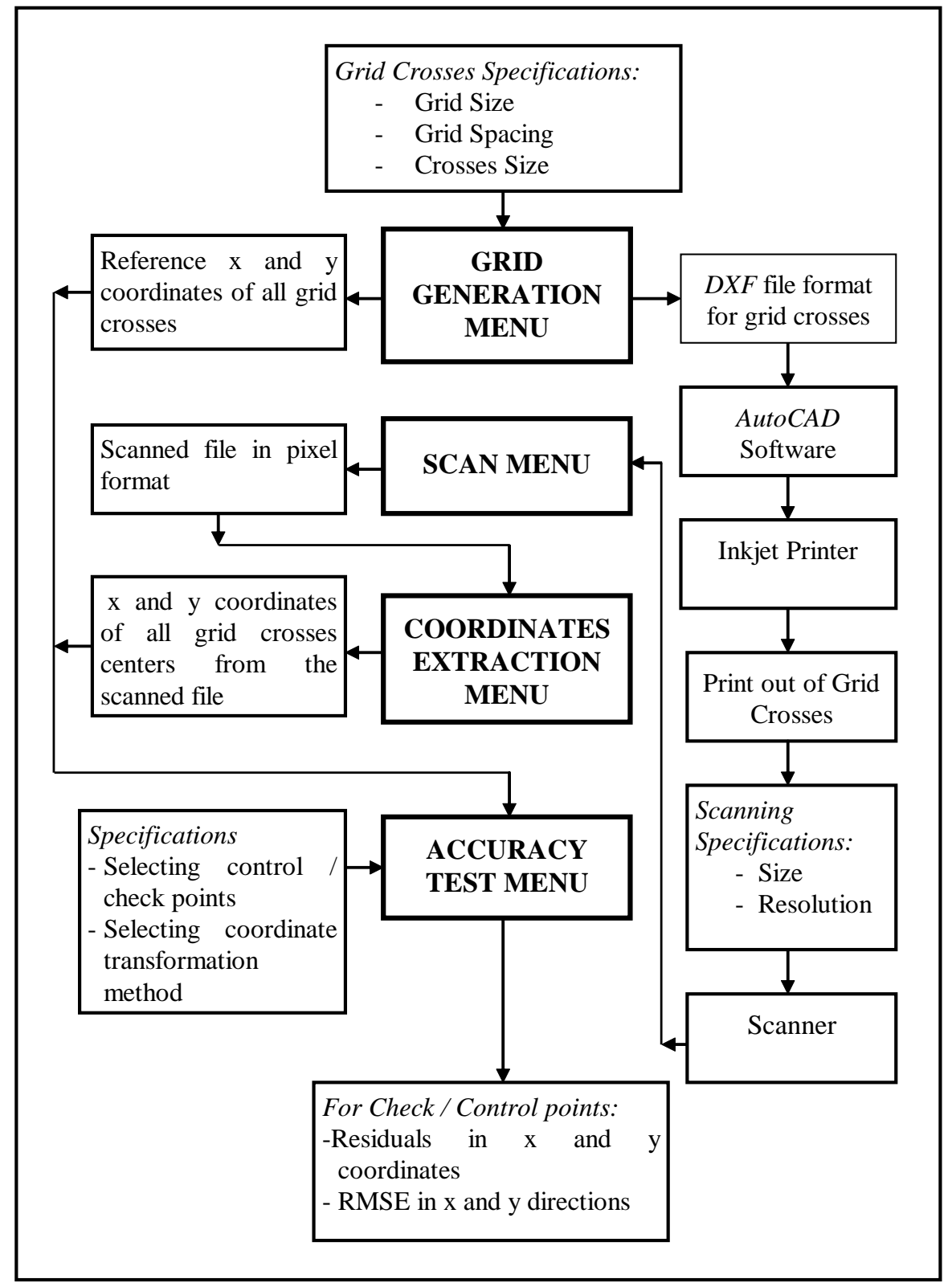

Figure 1. ScanCal Main Functions

- Computing the $\mathrm{x}, \mathrm{y}$ coordinates of each cross center as follows [8]:

$x=\frac{1}{n} \sum_{i=1}^{i=n} x_{i}, y=\frac{1}{n} \sum_{i=1}^{i=n} y_{i}$

where $\quad \mathrm{x}, \mathrm{y} \quad$ are the coordinates (in pixel) of the cross center;

$x_{i}, y_{i}$ are the $\mathrm{x}, \mathrm{y}$ image coordinates of pixel $\mathrm{i}$; and

$\mathrm{n} \quad$ is the total number of pixels forming the cross.

- Storing the $\mathrm{x}, \mathrm{y}$ image coordinates of all grid cross centers in specified ASCII file format for later use. 
International Journal of Advances in Scientific Research and Engineering (ijasre), Vol 8 (1), January -2022

\subsection{Geometric Accuracy Menu}

This menu reads the reference $\mathrm{x}$, y coordinates (in $\mathrm{mm}$ ) of the grid crosses as generated by Grid Generation menu and the measured x, y coordinates (in pixel) as extracted by Coordinates Extraction menu and performs coordinate transformation(s) for getting the necessary statistical data.

Points are classified as:

- Control points: These points are used for getting the parameters of specified coordinate transformation.

- Check points: These points are used for checking the discrepancy between the calculated $\mathrm{x}, \mathrm{y}$ coordinates of the crosses centers ( after getting the values of transformation parameters) and the reference ones for obtaining the necessary statistical data.

This menu enables the user to select the number and location of the control points which may be four or eight corner points (similar to the fiducial marks in aerial photographs), outer borderlines points or all points.

ScanCal software has the capability of performing the following $\mathrm{x}, \mathrm{y}$ coordinate transformation [12]:

- Affine transformation

The mathematical model is:

$$
\begin{aligned}
& x_{c}=a_{1} x_{m}+b_{1} y_{m}+c_{1} \\
& y_{c}=a_{2} x_{m}+b_{2} y_{m}+c_{2}
\end{aligned}
$$

where $x_{c}, y_{c}$ are the corrected coordinates (in $\mathrm{mm}$ ) of the control points;

$x_{m}, y_{m}$ are the measured coordinates (in pixel) of the control points; and

$a_{1}, \ldots \ldots, c_{2} \quad$ are the six parameters for the coordinate transformation.

At least three control points are required for obtaining the values of the transformation parameters.

- Projective transformation

The derivation of this coordinate transformation is shown in [12] and can be written as:

$$
\begin{aligned}
& x_{c}=\frac{a_{1} x_{m}+b_{1} y_{m}+c_{1}}{a_{o} x_{m}+b_{o} y_{m}+1.0} \\
& y_{c}=\frac{a_{2} x_{m}+b_{2} y_{m}+c_{2}}{a_{o} x_{m}+b_{o} y_{m}+1.0}
\end{aligned}
$$

in which $x_{c}, y_{c}$

are the corrected coordinates (in $\mathrm{mm}$ ) of the control points;

$$
x_{m}, y_{m}
$$

are the measured coordinates (in pixel) of the control points;

and

$$
a_{o}, \ldots \ldots, c_{2} \quad \text { are the eight parameters for the coordinate transformation. }
$$

In this case, the minimum number of control points for obtaining the values of the transformation parameters is four points.

- General two dimensional polynomial transformation (Polynomial transformation)

The mathematical model in this case is:

$$
\begin{aligned}
& x_{c}=a_{o}+a_{1} x_{m}+a_{2} y_{m}+a_{3} x_{m} y_{m}+a_{4} x_{m}^{2}+a_{5} y_{m}^{2} \\
& y_{c}=b_{o}+b_{1} x_{m}+b_{2} y_{m}+b_{3} x_{m} y_{m}+b_{4} x_{m}^{2}+b_{5} y_{m}^{2}
\end{aligned}
$$

where $x_{c}, y_{c} \quad$ are the corrected coordinates (in $\mathrm{mm}$ ) of the control points;

$$
\begin{aligned}
& x_{m}, y_{m} \quad \text { are the measured coordinates (in pixel) of the control points; and } \\
& a_{o}, \ldots \ldots, b_{5} \quad \text { are the twelve parameters for the coordinate transformation. }
\end{aligned}
$$

At least six control points are necessary for obtaining the values of the transformation parameters. 
For all methods of coordinate transformations, the software performs Least Squares Technique [11] for getting the values of the transformation parameters and statistical data such as:

- Computing the residuals (v) of $\mathrm{x}$, y coordinates of the grid crosses as follows:

- computing the corrected $\mathrm{x}$ and $\mathrm{y}$ coordinates using the parameters of the specified coordinate transformation method and the extracted $\mathrm{x}$, y coordinates

- $v_{x}$ or $v_{y}=$ computed $\mathrm{x}$ or $\mathrm{y}$ coordinate - reference one

- $\quad$ Computing the Root Mean Square Error (RMSE) values for the residuals of $x, y$ coordinates of the grid crosses for control, check and all points using:

$$
R M S E=\sqrt{\sum_{i=1}^{n}\left(v_{i}^{2}\right) / n}
$$

Where $\mathrm{n}$ is the number of points.

- Computing the Maximum Absolute Error (MAE) using:

$$
\text { Absolute Error }=|v|
$$

- Computing the Mean Error using:

$$
\text { Mean Error }=\frac{1}{n} \sum_{i=1}^{n} v_{i}
$$

Where $\mathrm{n}$ is the number of points.

Moreover, this menu has the capability of plotting the geometric scanner errors in the form of contours, vector of residuals, or three dimensions view. This capability is useful for studying the nature and effect of errors.

\subsection{Scanner Calibration Menu}

This menu performs automatically the steps of calibrating DTP scanners as described below.

- Transformation of the plate reference coordinates of the grid crosses to plate corrected pixel coordinate system The origin of the two coordinate systems is taken as the upper left grid cross. The corrected pixel coordinates of any grid cross can be obtained using:

$$
\begin{aligned}
& x_{1_{i}}=x_{i} \times \operatorname{Res} / 25.4 \\
& y_{1_{i}}=y_{i} \times \operatorname{Re} s / 25.4
\end{aligned}
$$

in which

$$
\begin{array}{ll}
x_{1_{i}}, y_{1_{i}} & \text { are the corrected coordinates of a grid cross in pixel; } \\
x_{i}, y_{i} & \text { are the plate coordinates of a grid cross in } \mathrm{mm} \text {; and } \\
\operatorname{Re} s & \text { is the scanning resolution in dpi. }
\end{array}
$$

(Some image processing softwares, such as CorelDraw [5], can perform this type of coordinates transformation by transforming DXF file format, as a vector format, to raster format).

- Transformation of the coordinates of the grid crosses from corrected pixel to corrected scanner coordinate systems

As shown in Figure 2, this transformation, is called linear conformal, includes simple translation and rotation of the corrected pixel coordinates system. The translation consists of shifting the origin of the corrected pixel coordinates system with $T_{x}$ and $T_{y}$ for x and y coordinates respectively. The rotation angle $\beta$ is produced due to improper placing of the plate on the scanner screen during scanning process. The rotation angle $\beta$ can be computed using the measured scanner coordinates of grid crosses of the upper row as:

$$
\beta=\frac{1}{n-1}\left(\sum_{i=2}^{n} \tan ^{-1}\left(\frac{y_{2_{1}}-y_{2_{i}}}{x_{2_{1}}-x_{2_{i}}}\right)\right)
$$


International Journal of Advances in Scientific Research and Engineering (ijasre), Vol 8 (1), January -2022

where $x_{2_{1}}, y_{2_{1}}$

$\mathrm{n}$ are the measured scanner coordinates of the upper left cross; and

is the number of grid crosses in the upper row

The corrected scanner coordinates of any grid cross can be obtained as follows [11]:

$$
\begin{aligned}
& x_{i}=T_{x}+x^{\prime}{ }_{i} \cos (\beta)+y_{i}{ }_{i} \sin (\beta) \\
& y_{i}=T_{y}-x^{\prime}{ }_{i} \sin (\beta)+y^{\prime}{ }_{i} \cos (\beta)
\end{aligned}
$$

in which $x_{i}, y_{i}$

are the corrected scanner coordinates in pixel of the grid cross;

$x_{i}^{\prime}, y^{\prime}{ }_{i}$ are the plate coordinates in pixel of the grid cross; and

$T_{x}, T_{y}$ are the measured scanner coordinates of the upper left grid cross.

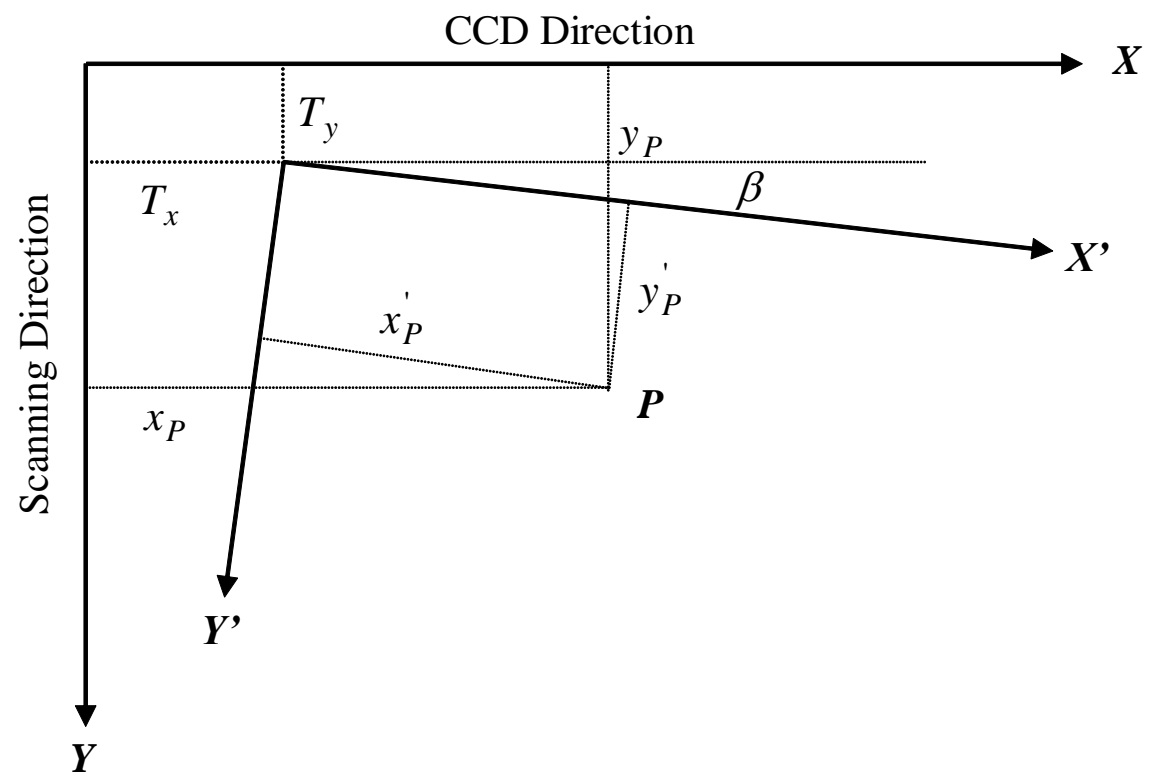

Figure 2. The Coordinate Systems for Linear Conformal Transformation

- Computation of the geometric corrections for the grid crosses

By comparing the corrected scanner coordinates (Equation 10) and the extracted scanner coordinates (Equation 1) of the grid crosses (both in pixel), the geometric corrections can be computed as:

$$
\begin{aligned}
& C_{x_{i}}=x_{c_{i}}-x_{m_{i}} \\
& C_{y_{i}}=y_{c_{i}}-y_{m_{i}}
\end{aligned}
$$

in which:

$$
\begin{array}{ll}
C_{x_{i}}, C_{y_{i}} & \text { the geometric corrections in pixel in x and y coordinates respectively of grid cross } \mathrm{i} \text {; } \\
x_{c_{i}}, y_{c_{i}} & \text { the } \mathrm{x} \text { and } \mathrm{y} \text { scanner corrected coordinates respectively of grid cross } \mathrm{i} \text {; and } \\
x_{m_{i}}, y_{m_{i}} & \text { the } \mathrm{x} \text { and y scanner extracted, or measured, coordinates of grid cross i respectively. }
\end{array}
$$

The computed geometric corrections reflect the effect of geometric errors without applying any errors minimizing or compensation method.

To increase the accuracy of getting the geometric corrections, the mean corrections for each grid cross of five scans taking with the same scanning resolution are computed and stored in suitable files.

The output of this step is the measured scanner coordinates of each grid cross with its geometric corrections. 
- Computation of the geometric corrections for each pixel in the scanner effective area

Interpolation method is used for computing the geometric corrections for any pixel locating between crosses. For this purpose, Surfer software is interfaced. The interfacing of Surfer software is performed using Surfer Automation Model method [9] without any interruption to the user. Out of the different methods of grid interpolation of Surfer software, Modified Shepard's method is recommended [7] and used in this research. The results of interfacing Surfer software are two files namely DataCal_X.grd and DataCal_Y.grd for specified scanning resolution. These files can be used for computing the geometric corrections in $\mathrm{x}$ and $\mathrm{y}$ extracted scanner coordinates of any pixel for new scan(s).

\subsection{Calibrating New Scan Menu}

This menu reads the extracted coordinates of the points of interest of the scanned image, computes their geometric corrections using the generated calibration files, as explained above, and computes their corrected $\mathrm{x}$ and y coordinates. The menu has options to perform different methods of coordinates transformation with displaying the necessary statistical data to test the validation of the calibration method. Desired control points number and pattern can be specified by the user of this software.

\section{TESTING THE PERFORMANCE OF ScanCAL}

This test consisted of testing the capabilities of the software for coordinates extraction and transformation. The basic steps for this purpose were:

- Generating the grid crosses to any specifications, using the capabilities of Grid Generation Menu, in the form of DXF file with the corresponding file of the reference $\mathrm{x}$ and $\mathrm{y}$ coordinates of grid crosses.

- Transforming the DXF file (in vector format) to pixel format at specified resolution by using the capabilities of CorelDraw software [5]. The generated pixel format files are similar to the scanned files but free-off any types of errors.

- Extracting the $\mathrm{x}$ and $\mathrm{y}$ coordinates of all grid crosses using the functions of Coordinates Extraction Menu.

- Applying all methods of coordinates transformation between the extracted $\mathrm{x}, \mathrm{y}$ coordinates and the reference coordinates using the available control points patterns $(4,8$, outer borderlines and all points).

- Computing the values of RMSE for each case.

The test showed that the values of RMSE are zero values in all cases and hence it can be concluded that the ScanCal software is functioning properly and gives desired accuracy.

\section{4- APPLICATION OF SCANCAL FOR TESTING AND CALIBRATING GEOMETRIC ERRORS OF LOW COST DTP SCANNER}

ScanCal was used to test and calibrating the geometric errors of VUEGO Scan Brisa Acer 640U scanner with A4 scan size.

The Grid Generation Menu was executed to generate the test pattern. This pattern consists of $19 * 19$ grid crosses (total of 361 crosses) with a grid spacing of $10 \mathrm{~mm}$. The AutoCAD software was used to print the generated grid crosses on HP transparency using inkjet printer(s). Laser printers were not used in this test to avoid their thermal effect which may damage the transparency.

The testing phase of low cost DTP scanner consisted of:

- $\quad$ Testing the plotting error

- $\quad$ Testing the Scan-to-Scan Error

- Testing the geometric accuracy of the scanner

- Geometric calibration of the scanner

- $\quad$ Testing the geometric calibration procedure

\subsection{Testing the plotting error}

One of the most important requirements for testing the geometric accuracy of the DTP scanners is using special manufactured glass plates containing the grid crosses [3].These plates are prepared by companies specializing in fine optics. After 
producing these plates, the coordinates of grid crosses are measured repeatedly at analytical plotter, or comparator, with reasonable accuracy for getting the reference coordinates of the grid crosses [3],[4]. The limitations of using this type of plates are costly, easy to damage, unavailable in all times, and unavailability of analytical plotter of suitable comparator.

Instead of using these plates, ScanCal prints the grid crosses on special type of HP transparency followed by plotting error test. The plotting error was tested by plotting the grid crosses using different types of inkjet printers. The print outs were scanned using different values of scan resolution. The computer and the scanner were turned off/on after each scan. The Coordinates Extraction menu was used for getting the measured $\mathrm{x}$ and $\mathrm{y}$ coordinates of all crosses from the scanned files. The six parameters of affine transformation were computed using the measured and reference coordinates of the grid crosses using all grid crosses as control points. The values of RMSE in $\mathrm{x}$ and $\mathrm{y}$ directions for each scan were computed and shown in Table 1. From Table 1, it is clear that the maximum difference in RMSE values of the three print outs is $2 \mu m$ which can be neglected. Finally, it can be concluded that the plotted grids are free off plotting error.

Table 1. Geometric Accuracy of the Scanner for Scans of Three Print outs of Different Printer Models

\begin{tabular}{|c|c|c|c|c|c|c|c|c|}
\hline \multirow[t]{2}{*}{ Printer Model } & \multicolumn{2}{|c|}{$\begin{array}{c}R M S E \text { Values } \\
\text { in } \mu m \\
(300 \text { dpi })\end{array}$} & \multicolumn{2}{|c|}{$\begin{array}{c}\text { RMSE Values } \\
\text { in } \mu m \\
\text { (600 dpi) }\end{array}$} & \multicolumn{2}{|c|}{$\begin{array}{c}R M S E \text { Values } \\
\text { in } \mu m \\
(900 \mathrm{dpi})\end{array}$} & \multicolumn{2}{|c|}{$\begin{array}{c}R M S E \text { Values } \\
\text { in } \mu m \\
(1200 \mathrm{dpi})\end{array}$} \\
\hline & $\mathbf{x}$ & $\mathbf{y}$ & $\mathbf{x}$ & $\mathbf{y}$ & $\mathbf{X}$ & $\mathbf{y}$ & $\mathbf{x}$ & $\mathbf{y}$ \\
\hline $\begin{array}{c}\text { HP Deskjet } \\
610 \mathrm{C}\end{array}$ & 67 & 32 & 66 & 29 & 66 & 29 & 66 & 29 \\
\hline $\begin{array}{c}\text { Epson Stylus } \\
\text { C64 Inkjet }\end{array}$ & 67 & 30 & 67 & 30 & 65 & 30 & 65 & 30 \\
\hline $\begin{array}{c}\text { Canon i560 } \\
\text { Inkjet }\end{array}$ & 68 & 31 & 66 & 30 & 66 & 30 & 66 & 30 \\
\hline
\end{tabular}

\subsection{Testing the Scan-to-Scan Error}

It was tested by taking five scans for the plotted grid crosses at scanning resolution of 300, 600, 900 and 1200 dpi. These scans were taking under the following conditions:

- Taking one scan, turning off/on the scanner and taking the next scan.

- Taking one scan, turning off/on the computer and scanner and taking the next scan.

- Taking one scan, turning off/on the computer and taking the next scan.

- Taking one scan followed by the next scan.

For this test, the coordinates of grid crosses were determined by Coordinates Extraction menu and the six parameters of affine transformation were computed using these values and the reference values using all grid crosses as control points. The values of RMSE for each scan were computed as shown in Table 2

Table 2 Geometric Accuracy of the Scanner for Different Scans

\begin{tabular}{|c|c|c|c|c|c|c|c|c|}
\hline \multirow[t]{2}{*}{$\begin{array}{c}\text { Scan } \\
\text { Number }\end{array}$} & \multicolumn{2}{|c|}{$\begin{array}{c}R M S E \text { Values } \\
\text { in } \mu m \\
\text { (300 dpi) }\end{array}$} & \multicolumn{2}{|c|}{$\begin{array}{c}\text { RMSE Values } \\
\text { in } \mu m \\
(600 \mathrm{dpi})\end{array}$} & \multicolumn{2}{|c|}{$\begin{array}{c}\text { RMSE Values } \\
\text { in } \mu \boldsymbol{m} \\
(900 \mathrm{dpi})\end{array}$} & \multicolumn{2}{|c|}{$\begin{array}{c}R M S E \text { Values } \\
\text { in } \mu m \\
(1200 \mathrm{dpi})\end{array}$} \\
\hline & $\mathbf{x}$ & $\mathbf{y}$ & $\mathbf{x}$ & $\mathbf{y}$ & $\mathbf{x}$ & $\mathbf{y}$ & $\mathbf{x}$ & $\mathbf{y}$ \\
\hline Scan 1 & 67 & 32 & 66 & 29 & 66 & 29 & 66 & 29 \\
\hline Scan 2 & 67 & 31 & 66 & 29 & 66 & 29 & 66 & 29 \\
\hline Scan 3 & 66 & 30 & 66 & 29 & 66 & 29 & 66 & 29 \\
\hline Scan 4 & 67 & 31 & 66 & 29 & 66 & 29 & 66 & 29 \\
\hline Scan 5 & 67 & 31 & 66 & 28 & 66 & 29 & 66 & 29 \\
\hline
\end{tabular}

From Table 2, it is clear that the maximum difference in RMSE is $1 \mu m$ which can be neglected and hence it can be concluded that the scanner is clear off scan-to-scan error. 
International Journal of Advances in Scientific Research and Engineering (ijasre), Vol 8 (1), January -2022

\subsection{Testing the Geometric Accuracy of the Scanner}

For this purpose, the grid crosses were scanned with scanning resolution of 300, 600, 900 and 1200 dpi. Five scans were taking for each scanning resolution. Coordinates

Extraction menu was used for automatic extraction of the $\mathrm{x}$ and $\mathrm{y}$ coordinates of grid crosses for each scan.

To simulate a realistic situation of aerial image scanning where only the fiducial marks are used for the interior orientation, four corner and eight (four corners and four points at the middle of the outer borderlines) points were used as control points.

Furthermore, the points at the outer borderlines were used as control points. Testing the global accuracy of the scanner was performed by choosing all points as control points [3].

In all cases of selected control point patterns, the remaining points were serving as check points.

For each scan and control point pattern, all methods of coordinate transformation were used, if applicable. The values of RMSE, MAE and Mean Errors in $\mathrm{x}$ and $\mathrm{y}$ directions for all points were computed. The mean of these values for the five scans were computed and tabulated in Table 3.

Table 3. Geometric Accuracy of the Scanner for Different Control Point Patterns and Methods of Coordinate Transformations

\begin{tabular}{|c|c|c|c|c|c|c|c|c|c|c|c|c|c|c|c|c|c|}
\hline \multirow{3}{*}{ 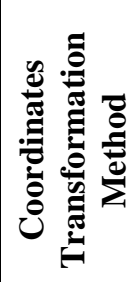 } & \multirow{3}{*}{ 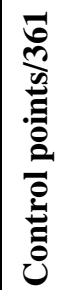 } & \multicolumn{4}{|c|}{300 dpi } & \multicolumn{4}{|c|}{600 dpi } & \multicolumn{4}{|c|}{900 dpi } & \multicolumn{4}{|c|}{1200 dpi } \\
\hline & & \multicolumn{2}{|c|}{$\begin{array}{l}R M S E \\
(\mu m)\end{array}$} & \multicolumn{2}{|c|}{$\begin{array}{c}M A E \\
(\mu m)\end{array}$} & \multicolumn{2}{|c|}{$\begin{array}{l}R M S E \\
(\mu m)\end{array}$} & \multicolumn{2}{|c|}{$\begin{array}{c}M A E \\
(\mu m)\end{array}$} & \multicolumn{2}{|c|}{$\begin{array}{l}R M S E \\
(\mu m)\end{array}$} & \multicolumn{2}{|c|}{$\begin{array}{c}M A E \\
(\mu m)\end{array}$} & \multicolumn{2}{|c|}{$\begin{array}{l}R M S E \\
(\mu m)\end{array}$} & \multicolumn{2}{|c|}{$\begin{array}{c}M A E \\
(\mu m)\end{array}$} \\
\hline & & $X$ & $\mathrm{Y}$ & $X$ & $\mathrm{Y}$ & $X$ & $\mathrm{Y}$ & $X$ & $\mathrm{Y}$ & $X$ & Y & $X$ & Y & $X$ & Y & $X$ & $\mathrm{Y}$ \\
\hline \multirow{4}{*}{ 尝 } & $4^{1}$ & 151 & 43 & 285 & 104 & 152 & 45 & 278 & 108 & 153 & 43 & 277 & 106 & 152 & 39 & 274 & 97 \\
\hline & $8^{2}$ & 120 & 43 & 248 & 109 & 122 & 43 & 241 & 108 & 119 & 42 & 236 & 110 & 118 & 39 & 234 & 108 \\
\hline & $72^{3}$ & 95 & 36 & 209 & 96 & 94 & 34 & 199 & 89 & 94 & 33 & 196 & 89 & 94 & 33 & 196 & 86 \\
\hline & $361^{4}$ & 67 & 31 & 207 & 107 & 66 & 29 & 205 & 99 & 66 & 29 & 210 & 91 & 66 & 29 & 199 & 89 \\
\hline \multirow{4}{*}{ 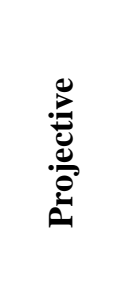 } & 4 & 165 & 40 & 304 & 99 & 166 & 43 & 297 & 103 & 167 & 40 & 296 & 94 & 166 & 36 & 292 & 85 \\
\hline & 8 & 116 & 44 & 242 & 115 & 117 & 43 & 236 & 115 & 114 & 42 & 229 & 118 & 113 & 41 & 227 & 118 \\
\hline & 72 & 88 & 38 & 201 & 103 & 87 & 37 & 191 & 101 & 86 & 36 & 187 & 102 & 86 & 36 & 187 & 104 \\
\hline & 361 & 53 & 43 & 166 & 148 & 53 & 41 & 164 & 142 & 52 & 42 & 170 & 138 & 52 & 42 & 162 & 136 \\
\hline \multirow{4}{*}{ 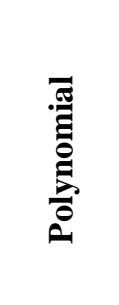 } & 4 & $--^{5}$ & -- & -- & -- & -- & -- & -- & -- & -- & -- & -- & -- & -- & -- & -- & -- \\
\hline & 8 & 64 & 41 & 161 & 99 & 65 & 37 & 158 & 93 & 58 & 39 & 140 & 95 & 56 & 39 & 139 & 98 \\
\hline & 72 & 39 & 32 & 102 & 80 & 39 & 30 & 87 & 72 & 39 & 30 & 82 & 71 & 37 & 30 & 85 & 72 \\
\hline & 361 & 33 & 27 & 95 & 75 & 32 & 25 & 90 & 64 & 32 & 25 & 81 & 61 & 31 & 25 & 78 & 64 \\
\hline
\end{tabular}

1 Four corner points

2 Four corners and four points at the middle of the outer borderlines

3 Points at outer borderlines

4 All points

5 Inapplicable

As depicted in Table 3, it can be concluded that:

- All plots show the systematic nature of errors distribution. 
- Increasing the scanning resolution has insignificant effect on improving the accuracy because the error sources and magnitude remain the same. In fact, with a higher scanning resolution, only the accuracy of coordinates extraction could be possibly improved but this gain would be very small compared to the whole error budget.

- Increasing the number of control points has significant effect on improving the accuracy.

- The errors are generally larger in $\mathrm{x}$ direction than in y direction. Increasing the number of the parameters of the coordinates transformation method reduces the errors in $\mathrm{x}$ direction better than in $\mathrm{y}$ direction. This reduction is significant when using the polynomial coordinate transformation method. This indicates that the large amount of the scanner geometric error is systematic.

- The maximum absolute errors are bounded and correspond to 2.5 - 3.5 RMSE.

- The global geometric errors of the scanner, RMSE values when using all points as control points [3], are 33 and $27 \mu \mathrm{m}$ in $\mathrm{x}$ and $\mathrm{y}$ direction respectively. These RMSE values are not suitable for photogrammetric applications which need at least geometric error of $20 \mu \mathrm{m}$ or better [2]. Furthermore, the above mentioned scanner accuracy may be sufficient for some cartographic and GIS application [4].

\subsection{Geometric Calibration of the Scanner}

As has been explained earlier, the corrections $C_{x}$ and $C_{y}$ for each grid cross of each scan were computed. The average values of these corrections of the five scans of each scanning resolution were determined. Surfer software was interfaced to generate the calibration files, DataCal_X.grd and DataCal_Y.grd, for each scanning resolution to predicate the corrections for any pixel locating within the scanner effective area.

To check the results of the calibration procedure, each scan was calibrated using the generated calibration files to find the corrections for each grid cross and its corrected $\mathrm{x}$ and $\mathrm{y}$ coordinates. The different methods of coordinates transformation were applied and RMSE, MAE and Mean Error values at check points were computed. The results of the five scans for each scanning resolution were similar and the average is shown in Table 4. From Tables 3 and 4, the following conclusions can be drawn:

Table 4. Geometric Accuracy of the Scanner after Calibration for Different Control Point Patterns and Methods of Coordinate Transformations

\begin{tabular}{|c|c|c|c|c|c|c|c|c|c|c|c|c|c|c|c|c|c|}
\hline \multirow{3}{*}{ 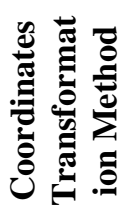 } & \multirow{3}{*}{ 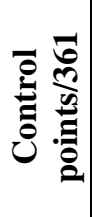 } & \multicolumn{4}{|c|}{300 dpi } & \multicolumn{4}{|c|}{600 dpi } & \multicolumn{4}{|c|}{900 dpi } & \multicolumn{4}{|c|}{1200 dpi } \\
\hline & & \multicolumn{2}{|c|}{$\begin{array}{l}\text { RMSE } \\
(\mu m)\end{array}$} & \multicolumn{2}{|c|}{$\begin{array}{c}M A E \\
(\mu m)\end{array}$} & \multicolumn{2}{|c|}{$\begin{array}{l}R M S E \\
(\mu m)\end{array}$} & \multicolumn{2}{|c|}{$\begin{array}{c}M A E \\
(\mu m)\end{array}$} & \multicolumn{2}{|c|}{$\begin{array}{l}\text { RMSE } \\
(\mu m)\end{array}$} & \multicolumn{2}{|c|}{$\begin{array}{c}M A E \\
(\mu m)\end{array}$} & \multicolumn{2}{|c|}{$\begin{array}{l}\text { RMSE } \\
(\mu m)\end{array}$} & \multicolumn{2}{|c|}{$\begin{array}{c}M A E \\
(\mu m)\end{array}$} \\
\hline & & $\mathbf{X}$ & $\mathbf{Y}$ & $\mathbf{X}$ & $\mathbf{Y}$ & $\mathbf{X}$ & $\mathbf{Y}$ & $\mathbf{X}$ & $\mathbf{Y}$ & $\mathbf{X}$ & $\mathbf{Y}$ & $\mathbf{X}$ & $\mathbf{Y}$ & $\mathbf{X}$ & $\mathbf{Y}$ & $\mathbf{X}$ & $\mathbf{Y}$ \\
\hline \multirow{4}{*}{ 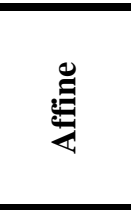 } & $4^{1}$ & 11 & 11 & 39 & 34 & 3 & 3 & 11 & 11 & 5 & 4 & 13 & 10 & 5 & 4 & 16 & 10 \\
\hline & $8^{2}$ & 11 & 10 & 41 & 30 & 3 & 3 & 10 & 11 & 4 & 4 & 12 & 10 & 4 & 3 & 15 & 10 \\
\hline & $72^{3}$ & 10 & 9 & 38 & 28 & 3 & 3 & 10 & 10 & 3 & 3 & 11 & 10 & 4 & 3 & 13 & 9 \\
\hline & $361^{4}$ & 10 & 9 & 39 & 29 & 3 & 3 & 10 & 9 & 3 & 3 & 11 & 9 & 4 & 3 & 13 & 9 \\
\hline \multirow{4}{*}{ 总 } & 4 & 11 & 13 & 40 & 39 & 4 & 3 & 12 & 11 & 4 & 3 & 13 & 10 & 5 & 5 & 17 & 13 \\
\hline & 8 & 11 & 10 & 42 & 33 & 3 & 3 & 11 & 11 & 4 & 3 & 13 & 10 & 4 & 3 & 13 & 10 \\
\hline & 72 & 10 & 9 & 38 & 28 & 3 & 3 & 10 & 10 & 3 & 2 & 11 & 9 & 4 & 3 & 13 & 9 \\
\hline & 361 & 10 & 9 & 38 & 28 & 3 & 3 & 10 & 9 & 3 & 2 & 11 & 9 & 4 & 3 & 13 & 9 \\
\hline \multirow{4}{*}{ 离 } & 4 & $--^{5}$ & -- & -- & -- & -- & -- & -- & -- & -- & -- & -- & -- & -- & -- & -- & -- \\
\hline & 8 & 13 & 12 & 48 & 34 & 3 & 3 & 10 & 11 & 5 & 3 & 14 & 11 & 4 & 3 & 14 & 11 \\
\hline & 72 & 10 & 9 & 39 & 29 & 3 & 3 & 10 & 10 & 3 & 3 & 11 & 9 & 3 & 3 & 13 & 9 \\
\hline & 361 & 10 & 9 & 38 & 28 & 2 & 2 & 9 & 9 & 3 & 3 & 10 & 9 & 3 & 3 & 13 & 9 \\
\hline
\end{tabular}

1 Four corner points

2 Four corners and four points at the middle of the outer borderlines

3 Points at outer borderlines

4 All points

5 Inapplicable

- The developed calibration procedure has significant effect on improving the accuracy of the scanner.

- The number of control points and the method of coordinates transformation have slight effect on the results.

This means that a large amount of the systematic errors has been minimized. 
- Scanning resolution more than 300 dpi has significant effect on improving the scanner accuracy. This may be due to improving the accuracy of coordinates extraction for the higher scanning resolution.

- The best scanning resolution, for the tested scanner, is $600 \mathrm{dpi}$.

\subsection{Testing the Geometric Calibration Procedure}

To check the validity of the calibration procedure, a pattern of $10 * 10$ grid crosses (total of 100 crosses) with a grid spacing of $15 \mathrm{~mm}$ was generated, printed out on HP transparency and checked for plotting error as has been explained earlier. The pattern was scanned using resolution of 300, 600, 900 and 1200 dpi. Five scans were taken for each scanning resolution.

Each scan was calibrated using the previously generated calibration files of the same scanning resolution to compute the corrected $\mathrm{x}$ and $\mathrm{y}$ coordinates of each grid cross. Four and eight control point patterns were used to simulate the aerial photographs. Affine and projective transformation methods were used and values of RMSE, MAE and Mean Errors at check points were computed.

On the other hand, to show the effects of the developed calibration procedure, statistical values at check points without calibration were also computed. The results of the five scans for each scanning resolution were similar and the average is computed. All results are depicted in Tables 5 and 6. From these tables, it can be seen that:

Table 5. Geometric Accuracy of the Scanner without Calibration for New Scan

\begin{tabular}{|c|c|c|c|c|c|c|c|c|c|c|c|c|c|c|c|c|c|}
\hline \multirow{3}{*}{ 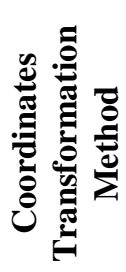 } & \multirow{3}{*}{ } & \multicolumn{4}{|c|}{300 dpi } & \multicolumn{4}{|c|}{$600 \mathrm{dpi}$} & \multicolumn{4}{|c|}{900 dpi } & \multicolumn{4}{|c|}{1200 dpi } \\
\hline & & \multicolumn{2}{|c|}{$\begin{array}{l}R M S E \\
(\boldsymbol{\mu m})\end{array}$} & \multicolumn{2}{|c|}{$\begin{array}{c}M A E \\
(\mu m)\end{array}$} & \multicolumn{2}{|c|}{$\begin{array}{l}\text { RMSEE } \\
(\boldsymbol{\mu M})\end{array}$} & \multicolumn{2}{|c|}{$\begin{array}{c}M A E \\
(\boldsymbol{\mu m})\end{array}$} & \multicolumn{2}{|c|}{$\begin{array}{l}\text { RMSE } \\
(\boldsymbol{\mu m})\end{array}$} & \multicolumn{2}{|c|}{$\begin{array}{c}M A E \\
(\mu m)\end{array}$} & \multicolumn{2}{|c|}{$\begin{array}{l}\text { RMSEE } \\
(\boldsymbol{\mu m})\end{array}$} & \multicolumn{2}{|c|}{$\begin{array}{c}\text { MAE } \\
(\boldsymbol{\mu m})\end{array}$} \\
\hline & & $\mathbf{X}$ & $\mathbf{Y}$ & $\mathbf{X}$ & $\mathbf{Y}$ & $\mathbf{X}$ & $\mathbf{Y}$ & $\mathbf{X}$ & $\mathbf{Y}$ & $\mathbf{X}$ & $\mathbf{Y}$ & $\mathbf{X}$ & $\mathbf{Y}$ & $\mathbf{X}$ & $\mathbf{Y}$ & $\mathbf{X}$ & $\mathbf{Y}$ \\
\hline \multirow{2}{*}{ 总 } & 4 & 25 & 19 & 64 & 51 & 24 & 24 & 60 & 52 & 24 & 21 & 56 & 46 & 24 & 19 & 50 & 47 \\
\hline & 8 & 25 & 19 & 61 & 50 & 22 & 24 & 56 & 50 & 24 & 18 & 53 & 43 & 24 & 18 & 49 & 49 \\
\hline \multirow{2}{*}{ 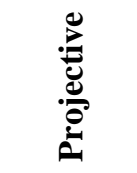 } & 4 & 26 & 19 & 67 & 53 & 25 & 24 & 62 & 54 & 24 & 22 & 60 & 48 & 24 & 22 & 55 & 48 \\
\hline & 8 & 24 & 19 & 61 & 51 & 23 & 22 & 56 & 49 & 24 & 17 & 52 & 43 & 24 & 18 & 50 & 48 \\
\hline
\end{tabular}

Table 6. Geometric Accuracy of the Scanner after Calibration for New Scan

\begin{tabular}{|c|c|c|c|c|c|c|c|c|c|c|c|c|c|c|c|c|c|}
\hline \multirow{3}{*}{ 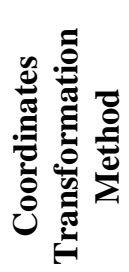 } & \multirow{3}{*}{ 总 } & \multicolumn{4}{|c|}{300 dpi } & \multicolumn{4}{|c|}{$600 \mathrm{dpi}$} & \multicolumn{4}{|c|}{900 dpi } & \multicolumn{4}{|c|}{1200 dpi } \\
\hline & & \multicolumn{2}{|c|}{$\begin{array}{l}R M S E \\
(\boldsymbol{\mu m})\end{array}$} & \multicolumn{2}{|c|}{$\begin{array}{c}M A E \\
(\boldsymbol{\mu} \boldsymbol{m})\end{array}$} & \multicolumn{2}{|c|}{$\begin{array}{l}R M S E \\
(\boldsymbol{\mu M})\end{array}$} & \multicolumn{2}{|c|}{$\begin{array}{c}M A E \\
(\boldsymbol{\mu m})\end{array}$} & \multicolumn{2}{|c|}{$\begin{array}{l}\text { RMSEE } \\
(\boldsymbol{\mu M})\end{array}$} & \multicolumn{2}{|c|}{$\begin{array}{c}M A E \\
(\mu m)\end{array}$} & \multicolumn{2}{|c|}{$\begin{array}{l}R M S E \\
(\mu m)\end{array}$} & \multicolumn{2}{|c|}{$\begin{array}{c}M A E \\
(\mu m)\end{array}$} \\
\hline & & $\mathbf{X}$ & $\mathbf{Y}$ & $\mathbf{X}$ & $\mathbf{Y}$ & $\mathbf{X}$ & $\mathbf{Y}$ & $\mathbf{X}$ & $\mathbf{Y}$ & $\mathbf{X}$ & $\mathbf{Y}$ & $\mathbf{X}$ & $\mathbf{Y}$ & $\mathbf{X}$ & $\mathbf{Y}$ & $\mathbf{X}$ & $\mathbf{Y}$ \\
\hline \multirow{2}{*}{$\stackrel{\Xi}{Z}$} & 4 & 20 & 14 & 56 & 37 & 4 & 4 & 13 & 12 & 6 & 6 & 16 & 18 & 6 & 4 & 14 & 13 \\
\hline & 8 & 17 & 13 & 49 & 38 & 4 & 4 & 12 & 11 & 6 & 6 & 17 & 17 & 4 & 4 & 10 & 13 \\
\hline \multirow{2}{*}{ 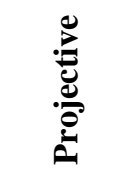 } & 4 & 22 & 15 & 59 & 42 & 4 & 4 & 13 & 12 & 6 & 6 & 16 & 17 & 6 & 4 & 16 & 12 \\
\hline & 8 & 17 & 14 & 49 & 41 & 4 & 4 & 13 & 12 & 6 & 6 & 16 & 17 & 4 & 4 & 9 & 12 \\
\hline
\end{tabular}

- The achieved geometric accuracy is 4-6 $\mu \mathrm{m}$ which is comparable to the accuracy of photogrametric scanners. 


\section{CONCLUSION}

The developed software provides a real time solution for testing the geometric accuracy and calibrating the DTP scanners without using manufactured plates which are costly and unavailable in all times. Furthermore, the software is also suitable for the manufactured plates if these plates are available.

ScanCal attains high degree of automation in extracting the image coordinates, data processing and calibration of the scanner with/without the user interaction.

The software is cost effective because it depends mainly on:

- using the available inkjet printers or pen plotters for taking hard copy of the calibrated grid crosses,

- simple hardware requirements, and

- interfacing the available softwares.

The developed software, and hence its built-in data processing methodology, can be used for testing and calibrating scanners of any size. Moreover, its application fields can be widened to include testing and calibrating other devices such as digitizing table.

The tested scanner is one of the cheapest DTP scanners which has a price of about $\$ 60$ US. Its geometric accuracy test shows that the low cost DTP scanners are unsuitable for photogrammetric applications but may be used in GIS-related applications. Using ScanCal software improves the geometric accuracy of these scanners to be 4-6 ${ }^{\mu m}$. These values of accuracy open the door-way for using low cost DTP scanners for photogrammetric applications.

ScanCal is quite versatile and provide affordable tool to the students and researchers in the universities and academic centres who are interested in the field of image processing or digital photogrammetry.

\section{REFERENCES}

[1] Autodesk, 2020. AutoCAD 19 Reference Manual. Autodesk, Inc., Sausalito, California, U.S.A.

[2] Baltsavias, E. P., 2004. Photogrammetric Scanners - Survey, Technological Developments and Requirements. Paper presented at the ISPRS Comm. I Symposium, 25-27 February, Bangalore, India. In IAPRS, Vol. 32, Part 1, pp. 44-52.

[3] Baltsavias, E. P., and Waegli, B., 2002. Quality Analysis and Calibration of DTP Scanners. Paper Presented at the 18 ISPRS Congress, 9-14 July, Vienna, Austria, In IAPRS, Vol. 31, Part B1, pp. 13-19.

[4] Baltsavias, E. P., and Patias, P., 2000. On the Use of DTP Scanners for Cartographic Applications. In Proc. Of the 17 International Cartographic Conference, 3-9 September, Barcelona, Spain, pp. 1179-1189.

[5] Corel Corporation , 2020. CorelDraw 2020 Reference Manual. Corel Corporation, Inc., CANADA.

[6] DVP-GS. 2007, Release 6.4. https://www.geospatialworld.net/news/dvp-gs-version-6-4-release/, Groupe ALTA, CANADA, accessed in December, 2020.

[7] El-Ashmawy, K., 2021. Mathematical Digital Terrain Model (DTM) Data for Testing DTM Generation Methodologies and Software, American Scientific Research Journal for Engineering, Technology, and Sciences (ASRJETS), Volume 76, No 1, pp 239-252

[8] Gonzalez, R. C., and Woods, R. E., 2018. Digital Image Processing, 6th Edition, Addison-Wesley, Inc., USA.

[9] Golden, 2018. Surfer 16 Reference Manual. Golden, Inc., Colorado, U.S.A.

[10] Gregory, K., 1998. Special Edition Using Visual C++ 6. Que, USA. 
International Journal of Advances in Scientific Research and Engineering (ijasre), Vol 8 (1), January -2022

[11] GHILANI, C. and WOLF, P., 2017. Adjustment Computations: Spatial Data Analysis, 6th Edition. JOHN WILEY \& SONS, INC., USA.

[12] Mikhail, E, James, S., and Chris, J., 2001. Introduction to Mordern Photogrammetry, John Wiley \& Sons,Inc.U.S.A.

[13] Schildt, H., 1992. Turbo C/C++: The Complete Reference, Second Edition. McGraw-Hill, Inc., USA. 